\title{
NUEVOS DATOS ICNOTAXONÓMICOS SOBRE Gyrolithes DEL PLIOCENO INFERIOR DE LA CUENCA DEL GUADALQUIVIR (LEPE, HUELVA, ESPAÑA)
}

\author{
Eduardo MAYORAL y Fernando MUÑIZ \\ Departamento de Geología. Facultad de Ciencias Experimentales. Universidad \\ de Huelva. 21819 Palos de la Frontera (Huelva).
}

\begin{abstract}
Mayoral, E. y Muñiz, F. 1998. Nuevos datos icnotaxonómicos sobre Gyrolithes del Plioceno Inferior de la Cuenca del Guadalquivir (Lepe, Huelva, España). [New ichnotaxonomic data about Lower Pliocene Gyrolithes from Guadalquivir Basin (Lepe, Huelva, Spain)]. Revista Española de Paleontología, 13 (1), 61-69. ISSN ()2136937.
\end{abstract}

\begin{abstract}
A new ichnospecies of Gyrolithes named Gyrolithes nodosus nov. ichnosp. is described from the marine Lower Pliocene silty-sandy facies in the SW sector of the Guadalquivir Basin (Lepe, Huelva, Spain). This ichnospecies is mainly characterized by a highly agglutinated linned wall with distinct pellets homogeneously arranged around it. Spiral diameter and interwhorl distance are decreasing in depth. Gyrolithes nodosus nov. ichnosp. is always associated to Ophiomorpha nodosa burrows, either in isolated forms or constituting an extension of this one.
\end{abstract}

Keywords: Trace fossils, Gyrolithes nodosus nov. ichnosp., Lower Pliocene, Guadalquivir Basin, Lepe, Huelva, Spain.

\section{RESUMEN}

Se describe una nueva icnoespecie de Gyrolithes, denominada Gyrolithes nodosus nov. ichnosp., en las facies limo-arenosas del Plioceno Inferior marino en el sector suroccidental de la Cuenca del Guadalquivir (Lepe, provincia de Huelva). Esta icnoespecie se caracteriza principalmente por poseer una pared con un fuerte revestimiento de carácter aglutinante y completamente peletizado. En profundidad, la estructura presenta una disminución paulatina en el diámetro espiral y en el espaciado intervuelta de las cámaras. Gyrolithes nodosus nov. ichnosp. aparece íntimamente asociada a Ophiomorpha nodosa, bien de forma aislada o formando parte de su trayectoria.

Palabras clave: Pistas fósiles, Gyrolithes nodosus nov. ichnosp., Plioceno Inferior, Cuenca del Guadalquivir, Lepe, Huelva, España.

\section{INTRODUCCIÓN}

Los materiales limo-arenosos del Neógeno superior en el sector SO de la Cuenca del Guadalquivir han sido objeto en los últimos años de estudios paleoicnológicos muy concretos (Mayoral y Muñiz, 1993; Mayoral y Muñiz, 1995; Muñiz et al., 1995; Muñiz y Mayoral, 1996), en especial los referidos a Gyrolithes, tanto desde el punto de vista icnotaxonómico como paleoetológico.

Nuevas observaciones realizadas en los materiales anteriores han permitido dar a conocer una icnoespecie nueva de Gyrolithes y aportar datos relacionados con aspectos paleoetológicos y paleoambientales.

\section{MATERIAL ESTUDIADO}

Los ejemplares estudiados proceden de un yacimiento situado unos $2 \mathrm{Km}$ al oeste de la localidad de Lepe (Huelva), en las inmediaciones del paraje conocido como "Cabezo del Tío Parra” (Fig. 1).

Desde el punto de vista geológico, las facies marinas siliciclásticas del Neógeno superior /Mioceno Superior (Tortoniense superior-Messiniense)-Plioceno Inferior; Mayoral y Muñiz, 1994], se depositaron en esta área de la Cuenca del Guadalquivir en discordancia sobre el sustrato Paleozoico (Carbonífero Inferior), representado principalmente por pizarras en facies $C u l m$ y que afloran unos $7 \mathrm{Km}$ 


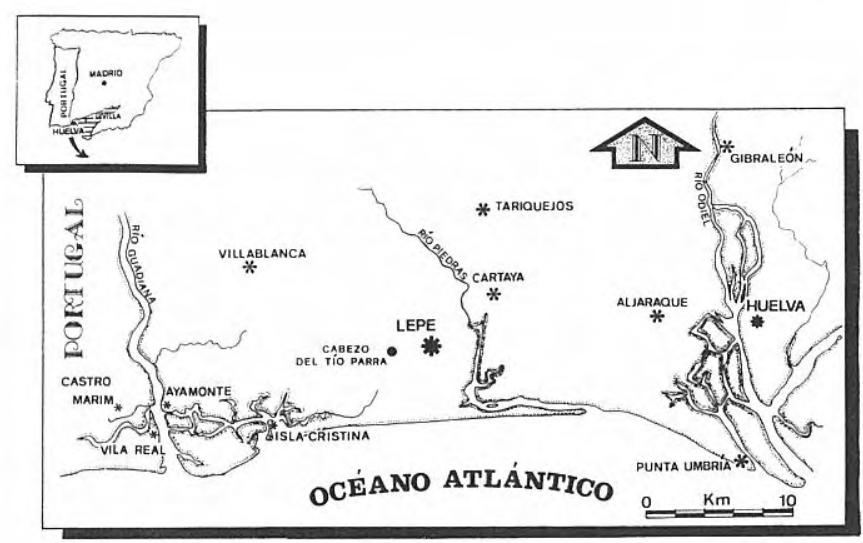

Figura 1. Localización geográfica de la zona estudiada.

al norte de la zona de estudio. A su vez, por encima de los materiales neógenos se apoyan otros relacionados con distintos niveles de terrazas fluviales de edad Cuaternaria (Pleistoceno Inferior) (Cáceres Puro, 1995). Éstos se disponen en discordancia sobre los materiales infrayacentes, ya sean del Plioceno Inferior, Mioceno Superior o Carbonífero (Fig. 2). La serie litoestratigráfica local se representa en el perfil de la figura 3. Dicha serie consta de muro hacia techo de los siguientes tramos:

Tramo 1: paquete de arenas gruesas y gravas, amarillentas, de unos $5 \mathrm{~m}$ de espesor, que se caracterizan por la presencia de una malacofauna abundante (bivalvos, gasterópodos, escafópodos), así como por cirrípedos balanomorfos, pinzas de crustáceos decápodos, restos de cetáceos (vértebras y costillas principalmente) y diversas pistas fósiles.

Tramo 2: sobre los materiales anteriores, se dispone de forma concordante un paquete de 1,5 m de espesor de limos arenosos de colores blancos y anaranjados, estos últimos como producto de las tinciones de óxidos e hidróxidos de hierro. Su contenido fosilífero esta representado por bivalvos y pistas, principalmente.

El siguiente nivel (Tramo 3) lo constituyen unos $2 \mathrm{~m}$ de arenas finas-medias, amarillentas, intensamente bioturbadas, caracterizadas por la presencia dominante de $O$. nodosa y Gyrolithes nodosus nov. ichnosp. objeto de este trabajo y definido en este nivel por la cantidad de ejemplares y la calidad del afloramiento.

Por encima del tramo anterior se encuentra un paquete de

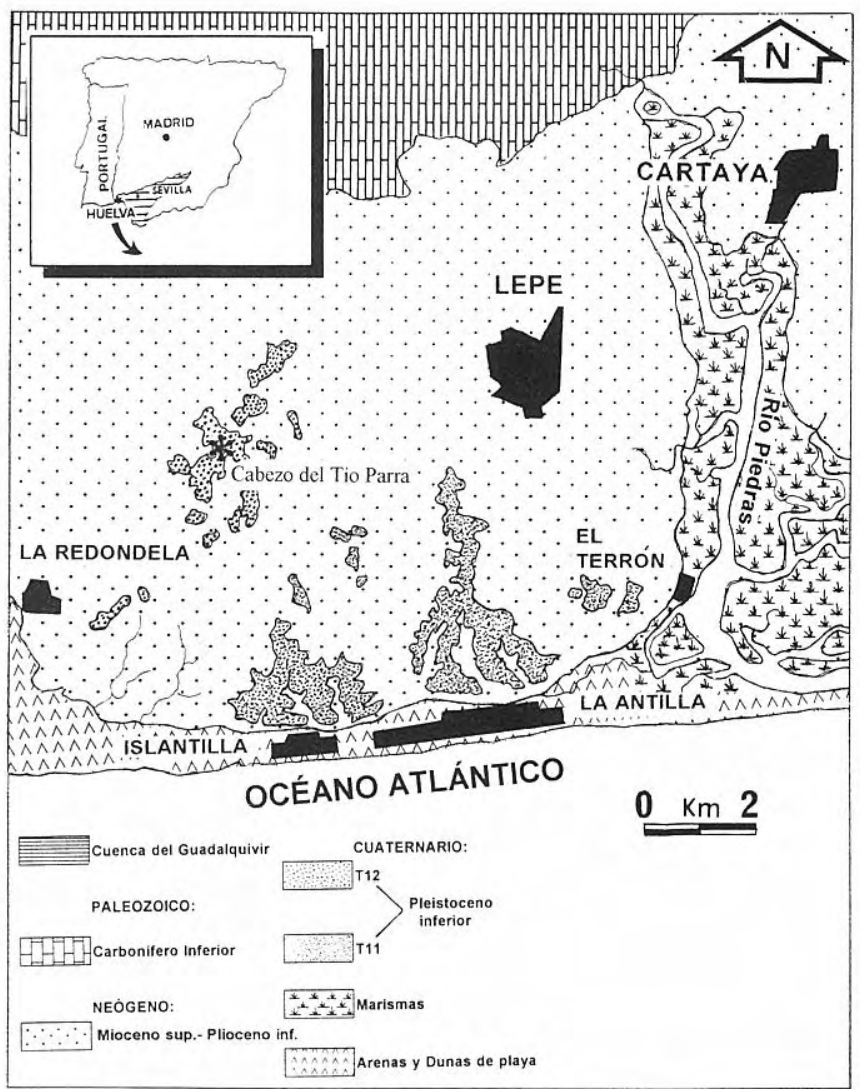

Figura 2. Contexto geológico del área de estudio.

unos $12 \mathrm{~m}$ de espesor (Tramo 4), formado por limos arenosos blancos, con laminacion paralela hacia techo, e intercalaciones de arenas medias-gruesas y niveles conglomeráticos de espesor decimétrico. Este tramo se caracteriza por presentar varias superficies ferruginosas de gran continuidad lateral, apreciable en algunos casos y que se asocian a superficies de no depósito y/u omisión-erosión, generalmente de carácter lumaquélico y en relación con horizontes intensamente bioturbados y bioerosionados (Mayoral y Muñiz, 1996).

El Tramo 5, de unos $4 \mathrm{~m}$ de espesor, está constituido en su parte inferior-media por una alternancia de arenas medias-gruesas, amarillentas, bioturbadas y niveles centimétricos de arcillas ricas en materia orgánica, también bioturbadas. La parte superior la forman unas arenas blanco-

\section{Lámina I}

1 Ejemplar de Gyrolithes nodosus nov. ichnosp. (Holotipo: LE16/Gn6) con enrollamiento dextrorso, en el que se observa claramente el carácter peletiforme de las paredes. Barra $=3 \mathrm{~cm}$.

2 Ejemplar de Gyrolithes nodosus nov. ichnosp. con enrollamiento sinestrorso (Paratipo: LE16/Gn2). Barra $=2 \mathrm{~cm}$.

3 Corte transversal de una sección espiral de Gyrolithes nodosus nov. ichnosp. (Paratipo: LE16/Gn16). Obsérvese el revestimiento peletiforme (flecha). Barra $=1 \mathrm{~cm}$.

4 Vista parcial de uno de los afloramientos con Gyrolithes nodosus nov. ichnosp. y Ophiomorpha nodosa. Escala $=5 \mathrm{~cm}$.

5 Detalle de Ophiomorpha nodosa Lundgren, asociada al horizonte de Gyrolithes nodosus nov. ichnosp. Se observan netamente los moldes externos de los pelets poligonales que conforman el revestimiento de las paredes. Escala $=1,7 \mathrm{~cm}$.

6 Detalle de la yuxtaposición entre Gyrolithes nodosus nov. ichnosp. (Paratipo: LE16/Gn4) y Ophiomorpha nodosa Lundgren.

7 Sistema intergradacional formado por Thalassinoides ichnosp. indet.- Gyrolithes variabilis (Mayoral y Muñiz, 1995) y Ophiomorpha nodosa Lundgren. Barra $=10 \mathrm{~cm}$. 
Lámina I
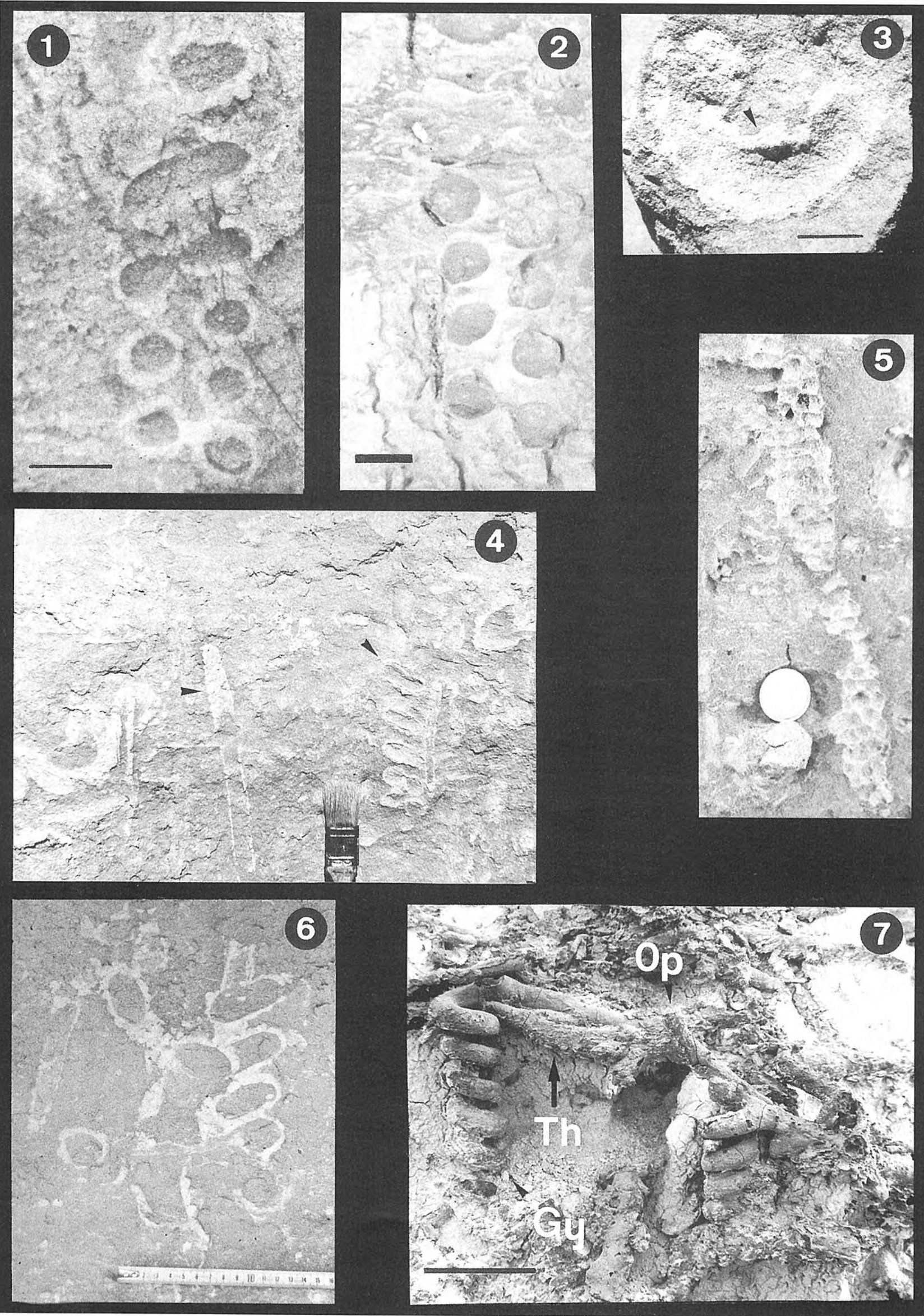

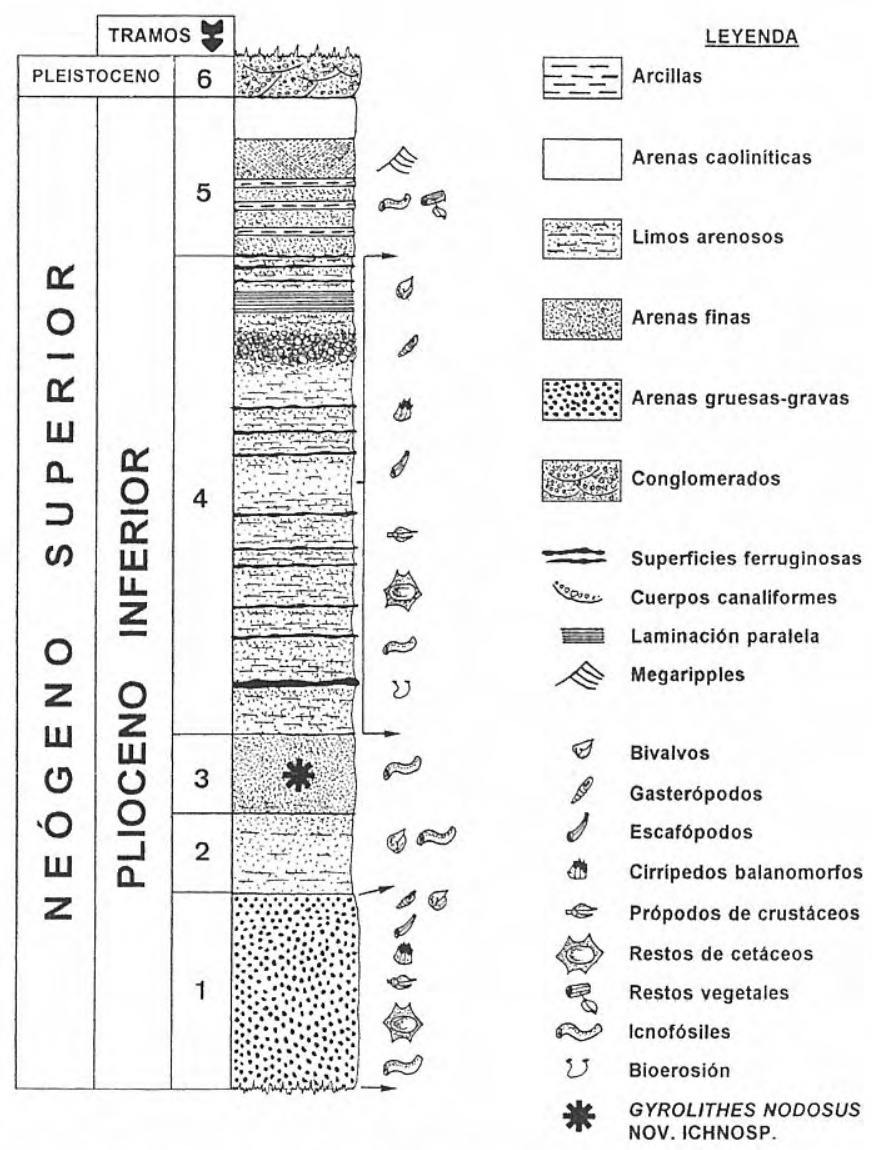

Figura 3. Sección LE 16. Columna litoestratigráfica local y situación del horizonte con Gyrolithes nodosus nov. ichnosp.

amarillentas con megaripples de escala métrica y unas arcillas caoliníticas con un espesor próximo a un metro.

Por último, la serie culmina con un paquete de 1-1,5 m de espesor (Tramo 6), de microconglomerados pardo-rojizos de cantos silíceos, que hacia techo presentan sets con laminación cruzada y fondo curvo.

\section{ICNOLOGÍA SISTEMÁTICA}

Icnogénero Gyrolithes de Saporta, 1884

Lista completa de sinonímia en Häntzschel, 1962, W 200.

Icnoespecie tipo: Gyrolithes davreuxi de Saporta, 1884; por designación subsecuente (Häntzschel, 1962, p. W200).

Diagnosis enmendada (en este trabajo):

Madrigueras más o menos verticalizadas en el sedimento, constituidas por una espiral circular que se enrolla indistintamente a la izquierda o a la derecha; la pared exterior puede presentar un revestimiento total o parcialmente peletizado con o sin marcas; el diámetro espiral y el espaciado intervuelta pueden o no variar simultáneamente desde su primera vuelta hasta la última. El radio de la vuelta espiral y el diámetro de la madriguera son bastante constantes y se pueden ramificar o interconectar con otras pistas, normalmente Thalassinoides

\begin{tabular}{|c|c|c|c|c|c|c|c|c|c|}
\hline \multicolumn{1}{c|}{} & $\begin{array}{c}\mathrm{Dg} \\
(\mathrm{cm})\end{array}$ & $\begin{array}{c}\mathrm{Hg} \\
(\mathrm{cm})\end{array}$ & $\begin{array}{c}\mathrm{Ev} \\
(\mathrm{cm})\end{array}$ & $\begin{array}{c}\mathrm{De} \\
(\mathrm{cm})\end{array}$ & $\alpha_{v}$ & $\alpha \mathrm{h}$ & $\begin{array}{c}\mathrm{Rv} \\
(\mathrm{cm})\end{array}$ & $\begin{array}{c}\theta p \\
(\mathrm{~cm})\end{array}$ & $\begin{array}{c}\mathrm{L} \\
(\mathrm{cm})\end{array}$ \\
\hline $\begin{array}{c}\text { VALORES } \\
\text { MEDIOS }\end{array}$ & 4,82 & 1,7 & 0,68 & 3,87 & $6,87^{\circ}$ & $19,6^{\circ}$ & 0,33 & 0,33 & 11,93 \\
\hline
\end{tabular}

\section{LEYENDA:}

Dg- diámetro de la sección de la galería. $\mathrm{Hg}$ - altura de la sección de la galería. Ev- espaciado intervuelta

De- diámetro espiral.

$\alpha_{v}$-ángulo de la desviación vertical del eje de enrollamiento.

$\alpha_{\text {h- }}$ ángulo de inclinación de las cámaras respecto a la horizontal.

Rv- revestimiento.

$\theta \mathrm{p}$ - diámetro de los pelets.

L- Longitud.

Tabla 1. Valores medios de Gyrolithes nodosus nov. ichnosp. según los parámetros morfológicos definidos por Mayoral (1986).

u Ophiomorpha formando sistemas intergradacionales más o menos complejos.

\section{Observaciones}

El icnogénero Gyrolithes se encuentra bien documentado en la bibliografía (ver Mayoral y Muñiz, 1993). Su amplia distribución abarca desde el Cámbrico Inferior [Formación Torreárboles en Fuente de Cantos; Badajoz, España (Liñán, 1984)] hasta el Holoceno de Florida, California, Carolina del Norte (EE.UU), México (Powell, 1977), Uruguay (Verde, M., comunicación personal) y Brasil (Dworschak and Rodrigues, 1997).

Hasta la fecha hay descritas nueve icnoespecies de Gyrolithes: G. davreuxi (de Saporta, 1884), G. marylandicus (Mansfield, 1927), G. clarki (Mansfield, 1930), G. mexicana (Mansfield, 1930), G. saxonicus (Häntzschel, 1934), G. bularti (Macsotay, 1967), G. polonicus (Fedonkin, 1980), G. vidali (Mayoral, 1986) y G. variabilis (Mayoral y Muñiz, 1995).

\section{Gyrolithes nodosus nov. ichnosp.}

Figs. 4, 6; Lám. I, figs. 1-4, 6.

1965 Xenohelix-like tunnels, Keij, 225, lám. 29, figs. 3-4. 1976 Gyrolithes-like spirals, Frey, Howard, Pryor, 207, fig. 6C.

Material tipo: Holotipo: LE16/Gn6 (Lám. I, fig. 1). Paratipos: LE16/Gn1 a LE16/Gn5, LE16/Gn7 a LE16/Gn16. La mayoría de los ejemplares se conservan in situ en los afloramientos, a excepción del holotipo y uno de los paratipos LE16/Gn5 (que se encuentran depositados en el Museo de Geología de la Universidad de Sevilla).

Localidad tipo: Lepe (Huelva). Al oeste del cabezo del Tío Parra, a lo largo del Arroyo Valleforero.

Estrato tipo: Tramo 3 de la sección LE16 de Lepe (Fig. 3).

Edad: Se sitúa aproximadamente unos $6 \mathrm{~m}$ por encima de un nivel muy próximo datado con nannoplancton calcáreo como Plioceno Inferior alto (Zancliense superior, Subzona CN 11b, Okada y Bukry, 1980; Cachão, comunicación 


\begin{tabular}{|c|c|c|c|c|c|c|}
\hline EDAD & AUTORES & ICNOESPECIE & Un. ESTRAT. & LOCALIDAD & Rv & PI \\
\hline PLIOCENO & $\begin{array}{l}\text { Mayoral, E. } \\
1986\end{array}$ & G. vidali & $\begin{array}{l}\text { Fm. Arcillas de } \\
\text { Gibraleón }\end{array}$ & $\begin{array}{l}\text { Palos de la Frontera } \\
\text { (Huelva)España }\end{array}$ & - & - \\
\hline MIOCENO & $\begin{array}{c}\text { Mansfield, W.C. } \\
1927\end{array}$ & G. marylandicus & Fm. St. Marys & $\begin{array}{l}\text { Maryland } \\
\text { (USA) }\end{array}$ & - & - \\
\hline MIOCENO & $\begin{array}{c}\text { Mansfield, W.C. } \\
1930\end{array}$ & G. clarki & Grupo Monterey & $\begin{array}{l}\text { California } \\
\text { (USA) }\end{array}$ & - & - \\
\hline MIOCENO & $\begin{array}{c}\text { Mansfield, W.C. } \\
1930\end{array}$ & G. marylandicus & Grupo Monterey & $\begin{array}{c}\text { California } \\
\text { (USA) }\end{array}$ & - & - \\
\hline MIOCENO & $\begin{array}{l}\text { Dryden, L. } \\
1933\end{array}$ & $\begin{array}{c}\text { Gyrolithes } \\
\text { ichnosp. indet. }\end{array}$ & Fm. Calvert & $\begin{array}{l}\text { Maryland } \\
\text { (USA) }\end{array}$ & - & - \\
\hline MIOCENO & $\begin{array}{l}\text { Kilpper, K. } \\
1962\end{array}$ & $\begin{array}{c}\text { Xenohelix } \\
\text { ichnosp. indet. }\end{array}$ & Fm. Braunkohlen & Alemania & $A-P i$ & Op. \\
\hline MIOCENO & $\begin{array}{l}\text { Keij, A.J. } \\
1965\end{array}$ & $\begin{array}{c}\text { Gyrolithes } \\
\text { ichnosp. indet. }\end{array}$ & Fm. Belait & Borneo & $A-P i$ & Op. \\
\hline MIOCENO & $\begin{array}{l}\text { Macsotay, } \mathrm{O} . \\
\quad 1967\end{array}$ & G. bularti & Fm. Cubagua & Venezuela & - & - \\
\hline MIOCENO & $\begin{array}{c}\text { Gernant, R.E. } \\
1970\end{array}$ & G. marylandicus & $\begin{array}{l}\text { Fm. Choptank } \\
\text { Mb. St. Leonard }\end{array}$ & $\begin{array}{l}\text { Maryland } \\
\text { (USA) }\end{array}$ & - & - \\
\hline MIOCENO & $\begin{array}{l}\text { Gernant, R.E. } \\
\quad 1971\end{array}$ & G. marylandicus & Fm. Calvert & $\begin{array}{c}\text { Maryland } \\
\text { (USA) }\end{array}$ & - & - \\
\hline MIOCENO & $\begin{array}{c}\text { Gernant, R.E. } \\
1972\end{array}$ & G. marylandicus & $\begin{array}{l}\text { Fm. St. Marys } \\
\text { Estrato } 22\end{array}$ & $\begin{array}{l}\text { Maryland } \\
\text { (USA) }\end{array}$ & - & - \\
\hline MIOCENO & $\begin{array}{c}\text { Muñiz, F. et al. } \\
1995\end{array}$ & $\begin{array}{c}\text { Gyrolithes } \\
\text { ichnosp. indet. }\end{array}$ & $\begin{array}{l}\text { "Limos blancos de } \\
\text { Lepe" }\end{array}$ & $\begin{array}{c}\text { Lepe (Huelva) } \\
\text { España }\end{array}$ & $A-P i$ & Th-Op \\
\hline MIOCENO & $\begin{array}{c}\text { Mayoral y Muñiz } \\
1995\end{array}$ & G. variabilis & $\begin{array}{l}\text { "Limos blancos de } \\
\text { Lepe" }\end{array}$ & $\begin{array}{c}\text { Lepe (Huelva) } \\
\text { España }\end{array}$ & $A-P i$ & Th-Op \\
\hline MIOCENO & $\begin{array}{l}\text { Christiansen y } \\
\text { Curran } 1995\end{array}$ & G. marylandicus & Fm. St. Mary's & $\begin{array}{l}\text { Maryland } \\
\text { (USA) }\end{array}$ & - & - \\
\hline OLIGOCENO & $\begin{array}{c}\text { Föllmi y Grimm } \\
1990\end{array}$ & $\begin{array}{c}\text { Gyrolithes } \\
\text { ichnosp. indet. }\end{array}$ & Fm. San Gregorio & $\begin{array}{c}\text { Baja California } \\
\text { (México) }\end{array}$ & - & Th. \\
\hline EOCENO & $\begin{array}{c}\text { Stenzel et al. } \\
\quad 1957\end{array}$ & $\begin{array}{c}\text { Xenohelix } \\
\text { ichnosp. indet. }\end{array}$ & "Stone city beds" & $\begin{array}{l}\text { Texas } \\
\text { (USA) }\end{array}$ & - & - \\
\hline EOCENO & $\begin{array}{c}\text { Stanton y Warme } \\
1971\end{array}$ & $\begin{array}{c}\text { Xenohelix } \\
\text { ichnosp. indet. }\end{array}$ & Fm. Stone City & $\begin{array}{l}\text { Texas } \\
\text { (USA) }\end{array}$ & A & On.? \\
\hline EOCENO & $\begin{array}{c}\text { Hester y Pryor } \\
1972\end{array}$ & $\begin{array}{l}\text { Xenohelix } \\
\text { marilandica }\end{array}$ & Fm. Tallahatta & $\begin{array}{c}\text { Mississippi State } \\
\text { (USA) }\end{array}$ & $A-P i$ & On. \\
\hline EOCENO & $\begin{array}{l}\text { King, M. } \\
1987\end{array}$ & $\begin{array}{l}\text { Gyrolithes } \\
\text { ichnosp. indet. }\end{array}$ & Fm. Dellmar & $\begin{array}{l}\text { California } \\
\text { (USA) }\end{array}$ & - & Op. \\
\hline
\end{tabular}

Tabla 2. Icnoespecies de Gyrolithes citadas en el Neógeno. Rv: Revestimiento: A: aglutinante, Pi: peletización irregular. PI: Pistas intergradacionales relacionadas: Th: Thalassinoides ichnosp. indet., Op: Ophiomorpha ichnosp. indet., On: Ophiomorpha nodosa. 


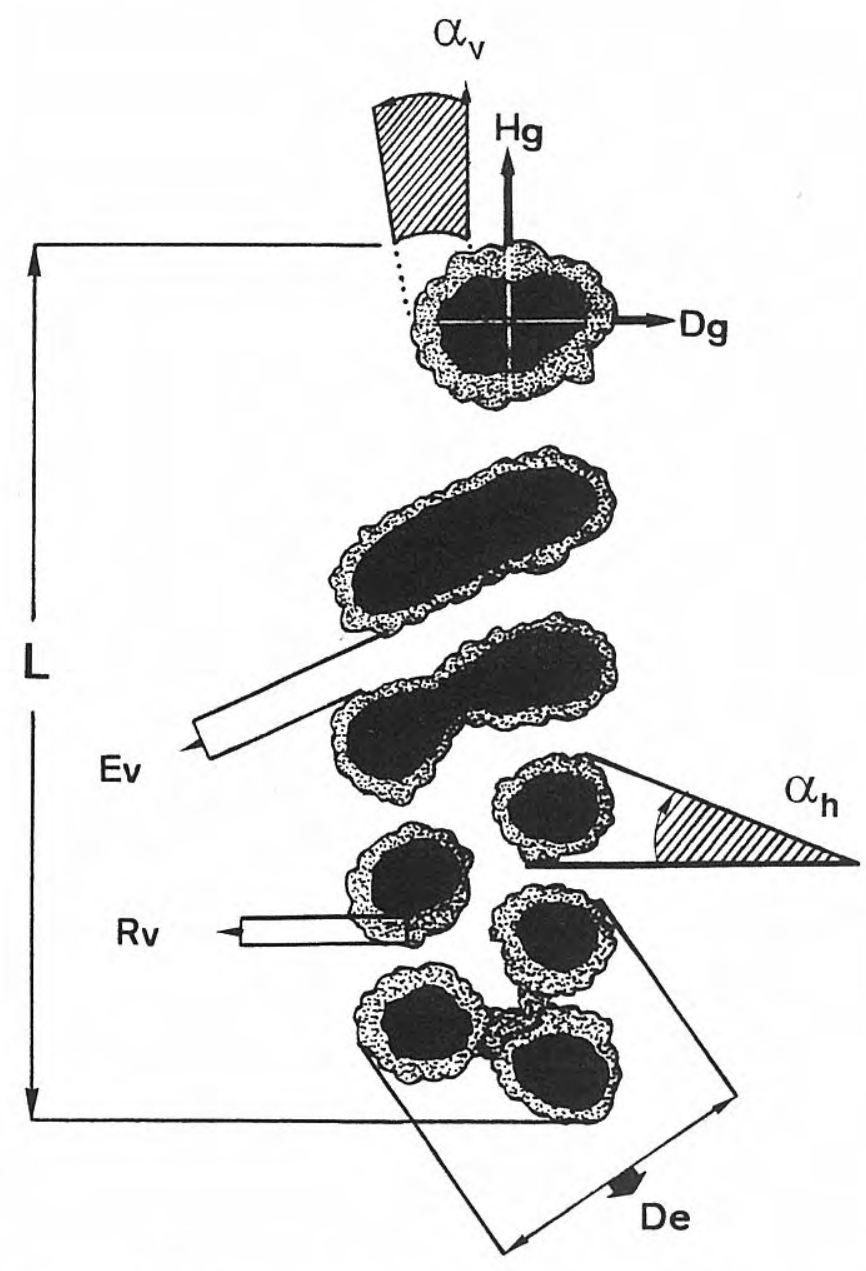

Figura 4. Parámetros morfológicos estudiados en Gyrolithes nodosus nov. icnosp. (de enrollamiento sinestrorso): De: diámetro espiral; Dg: diámetro de la sección de la galería; Ev: espaciado intervuelta; Hg: altura de la sección de la galería; Rv: grosor del revestimiento; $\alpha \mathbf{h}$ : ángulo de la inclinación de las cámaras respecto a la horizontal; $\alpha \mathbf{v}$ : ángulo de la desviación vertical del eje de enrollamiento; L: longitud.

personal), por lo que su edad podría establecerse al menos, como Plioceno Inferior.

Origen del nombre: del Latín nodosus, - a, -um: nudoso, lleno de nudos.

\section{Diagnosis}

Madriguera con un enrollamiento helicoidal, vertical, tanto dextrorso como sinestrorso, con su eje de giro ligeramente inclinado. Presenta una pared con un revestimiento aglutinante de carácter pelítico o limoso, con pelets muy bien diferenciados y repartidos de forma homogénea alrededor de todo el conjunto espiral. El diámetro espiral y el espaciado intervuelta tienden a disminuir simultáneamente conforme la estructura profundiza en el sedimento.

Spiral burrows describing a dextral or sinistral path with the coiling axis close to vertical position. It's characterized by a highly agglutinated linned wall with pellets homogeneously arranged around the whole spiral system. The relation between spiral diameter and interwhorl distance tends to decrease simultaneously in depth.

\section{Descripción}

Estructura que consiste en una espiral que se enrolla tanto en sentido dextrorso como sinestrorso (Lám. I, figs. 1, 2 ), con el eje de enrollamiento ligeramente desviado de la vertical $(\alpha v)$ unos $6,8^{\circ}$ de media y un ángulo medio de inclinación de las cámaras respecto a la horizontal $(\alpha \mathrm{h})$ de 19,6 (Tabla I; Fig. 5). La sección de las madrigueras es de subcircular a subelipsoidal con un diámetro (Dg) variable de 1 a $3,3 \mathrm{~cm}$ y una altura de la cámara (Hg) (Mayoral, 1986) de 0,8 a 2,5 cm. El valor medio de la longitud es de $11,9 \mathrm{~cm}$, con un número de vueltas máximo de 6 .

El diámetro espiral (que varía entre 3,3 y $4,6 \mathrm{~cm}$ ) y el espaciado intervuelta (que 1o hace entre 0,5 y $0,8 \mathrm{~cm}$ ) disminuyen ambos en sentido descendente, es decir, desde la primera vuelta de la madriguera hasta la última (Tabla 1; Figs. 4 y 5). Las paredes se presentan con un revestimiento (Gámez Vintaned y Liñán, 1996) claramente aglutinante, de carácter arcilloso o limoso, de 1 a $6 \mathrm{~mm}$ de grosor, con pelets muy netos y bien diferenciados distribuidos de forma homogénea alrededor de todo el sistema espiralado (Figs. 4 y 6; Lám. I, figs. 1-4, 6). El diámetro de los pelets varía de 2 a $4 \mathrm{~mm}$, con una media de $3,3 \mathrm{~mm}$ (Tabla 1 ).

El 90\% de las madrigueras presentan un relleno pasivo, de litología semejante a la del sedimento circundante (arenas finas-medias) y se encuentran conservadas como relieves completos, aislados (en secciones transversales y/o longitudinales Fig. 6; Lám. I, figs 1-2, 4 y 6), o en conexión con Ophiomorpha nodosa (Fig. 6; Lám. I, figs. 1, 4, 6). Las yuxtaposiciones entre ambos icnogéneros también suelen ser frecuentes (Lám. I, figs. 4 y 6).

\section{Discusión}

Ejemplares de Gyrolithes con paredes peletizadas ya han sido figurados y citados escuetamente en la bibliografía: Xenohelix (=Gyrolithes) ichnosp. indet. (Keij, 1965, p. 228, lám. 29, figs. 3-4); Xenohelix (=Gyrolithes) ichnosp. indet. (Hester y Pryor, 1972, p. 682, fig. 6); Gyrolithes ichnosp. indet. (Frey et al., 1978, p. 206, fig. 6c); y Gyrolithes variabilis (Mayoral y Muñiz, 1995, p.195, Lám. I, fig.5). La diferencia de todos ellos respecto a $G$. nodosus radica en que la presencia de pelets en los primeros es casual y su distribución muy irregular. En la mayoría de los Gyrolithes del Terciario (Tabla 2), lo habitual es que las paredes sean lisas (sin revestimiento) o que estén surcadas por marcas de rastrillaje (Gámez Vintaned, 1995), que discurren paralelas u oblicuas a la pendiente espiral de las cámaras, circunstancia ésta que no se ha detectado en nuestros ejemplares.

Al margen de esta diferencia esencial, las mayores similitudes se establecen con G. variabilis (morfotipo C; Mayoral y Muñiz, 1995) (Lám. I, fig. 7), no sólo por ocupar distribuciones areales y temporales próximas, sino por presentar una trayectoria en profundidad muy parecida. 

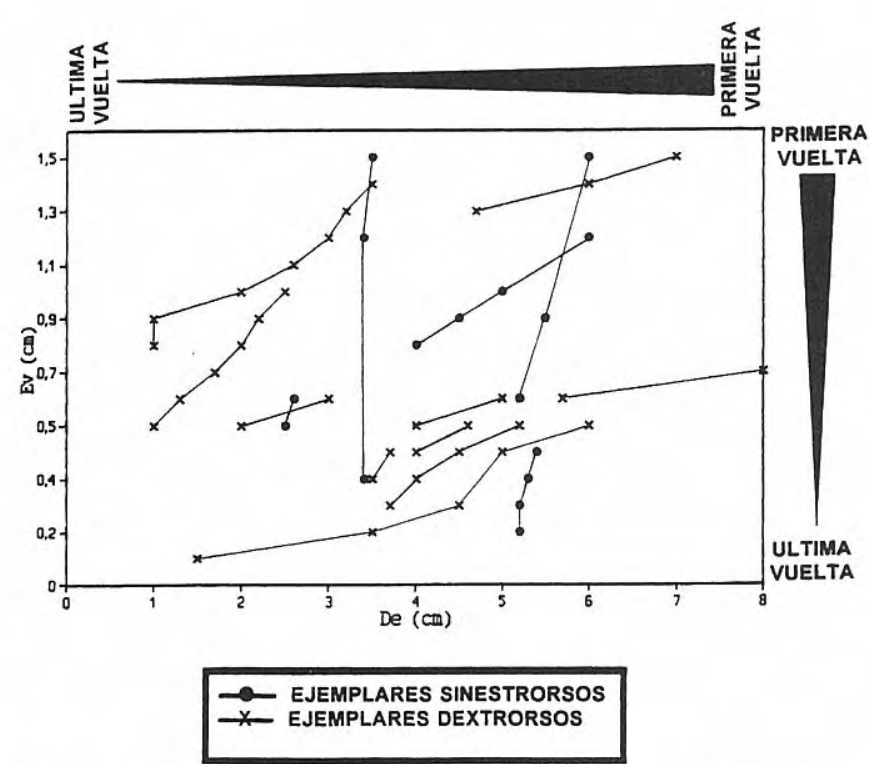

Figura 5. Relación entre el Diámetro espiral (De) y el Espaciado intervuelta (Ev) según el sentido descendente en los ejemplares de Gyrolithes nodosus nov. icnosp.

En ambas icnoespecies el porcentaje de reducción tanto del parámetro De como del Ev es similar, aunque más notable en $G$. variabilis (Tabla 3 ).

\section{Significado paleoetológico}

Gyrolithes nodosus nov. ichnosp. se interpreta como una estructura etológica (Gámez Vintaned y Liñán, 1996) independiente dentro de un sistema intergradacional junto a Ophiomorpha nodosa, resultado de la actividad excavadora de un artrópodo marino que sigue un patrón de desplazamiento espiral, descendente en la vertical (helicotáxico, según Gámez Vintaned y Liñán, 1996). Con esta trayectoria se favorece el máximo beneficio en la explotación del alimento (fodinichnia) con el mínimo gasto de desplazamiento. Al mismo tiempo, la naturaleza (arenas finas-medias) y consistencia del sustrato (probablemente con un alto contenido en agua) en un medio que se interpreta de energía media-alta, son

\begin{tabular}{|c|c|c|c|c|c|c|}
\cline { 2 - 7 } \multicolumn{1}{c|}{} & S/D & $\begin{array}{c}\text { De } \\
(\mathrm{cm})\end{array}$ & $\begin{array}{c}\% \text { Reducción } \\
\text { De }\end{array}$ & $\begin{array}{c}\text { Ev } \\
(\mathrm{cm})\end{array}$ & $\begin{array}{c}\% \text { Reducción } \\
\text { Ev }\end{array}$ & $\mathrm{Nv}$ \\
\hline $\begin{array}{c}\text { G. variabilis } \\
\text { "morfotipo C" }\end{array}$ & $\mathrm{S}$ & $7,1-5,2$ & $27-38 \%$ & $1,2-0,6$ & $50-55 \%$ & 4 \\
\cline { 2 - 7 } & $\mathrm{D}$ & $8-6,8$ & $3-15 \%$ & $1,9-1,4$ & $26-35 \%$ & 3 \\
\hline $\begin{array}{c}\text { G. nodosus } \\
\text { nov. ichnosp. }\end{array}$ & $\mathrm{S}$ & $6-4$ & $17-33 \%$ & $1,3-0,9$ & $8-30 \%$ & 4 \\
\cline { 2 - 7 } & $\mathrm{D}$ & $6-5,5$ & $2-9 \%$ & $1,5-0,7$ & $7-30 \%$ & 3 \\
\hline
\end{tabular}

\section{LEYENDA:}

D: enrollamiento dextrorso.

S: enrollamiento sinestrorso.

De: diámetro espiral.

Ev: espaciado intervuelta

Nv: número de vueltas.

Tabla 3. Tabla comparativa entre los porcentajes de reducción del Diámetro espiral (De) y el Espaciado intervuelta (Ev) para Gyrolithes variabilis y Gyrolithes nodosus nov. ichnosp. D: dextrorsos; S: sinestrorsos.

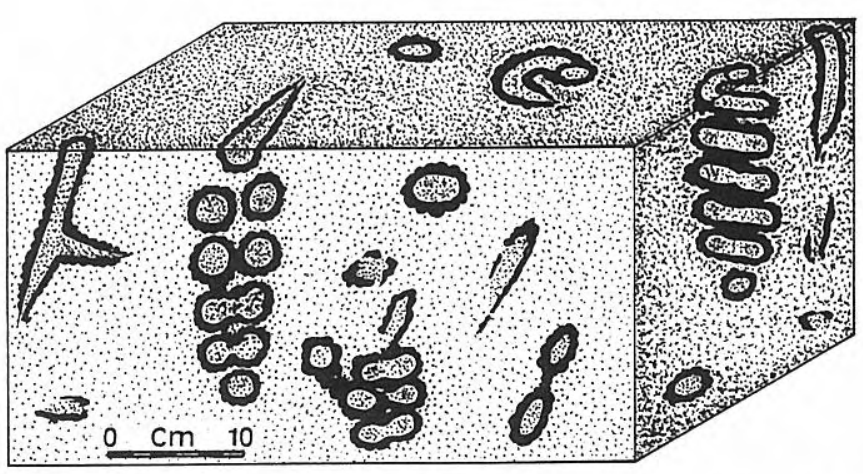

Figura 6. Bloque diagrama que ilustra las secciones longitudinales y transversales de Gyrolithes nodosus-Ophiomorpha nodosa y las yuxtaposiciones entre ambos.

factores que inducen a los productores a estabilizar permanentemente el sistema de madrigueras GyrolithesOphiomorpha (domichnia), mediante la adhesión uniforme a sus paredes de sedimento limoso retrabajado en forma de pelets. Esta acción podría ocultar o destruir cualquier marca de rastrillaje que pudiera haber dejado en el transcurso de la excavación; señales que por otra parte suelen ser muy frecuentes en este icnogénero.

\section{Organismos productores}

La bibliografía recoge una gran variedad de interpretaciones y de organismos relacionados con la producción de Gyrolithes: moldes de raíces o perforaciones de bivalvos (Barbour, 1892), gasterópodos (Woodward, 1922) o plantas (Mansfield, 1927); domicilio de roedores (Schultz, 1942); o de invertebrados de cuerpos blandos (Dryden, 1933), ya sean enteropneustos (Horst, 1940), anélidos poliquetos, como los actuales Notomastus lobatus Hartman y N. latericeus Sars (Powell, 1977) (Verde, M., comunicación personal) o anélidos en general (Beynon et al., 1988); o crustáceos decápodos (von Ammon, 1900; Häntzschel, 1934, 1962; Toots, 1963; Häntzschel, 1965; Keij, 1965; Gernant, 1972; Hester y Pryor, 1972; Bromley y Frey, 1974; Mayoral, 1986; Mayoral y Muñiz, 1993; Mayoral y Muñiz, 1995, Dworschak y Rodrigues, 1997, entre otros muchos).

Sin embargo, la existencia de sistemas de madrigueras con paredes peletizadas es típica de la actividad de crustáceos decápodos (Keij, 1965; Hester y Pryor, 1972; Bromley y Frey, 1974; Frey et al., 1978; Ekdale, 1992; Mapples y West, 1992; Muñiz et al., 1995). Este tipo de construcción se encuentra muy bien referido en los decápodos marinos actuales (Ekdale et al., 1984, p. 202204; Bromley, 1990, p. 71-90; Ekdale, 1992, p. 147-150; Mayoral y Muñiz, 1993, p. 20). Otro criterio que apoya esta hipótesis es la presencia de marcas de rastrillaje en las paredes, aunque en nuestros ejemplares no se han podido conservar por la causa comentada anteriormente. De cualquier forma, los caracteres morfológicos encontrados en Gyrolithes nodosus nov. ichnosp. parecen relacionarlo sin ninguna duda con la actividad 
excavadora de crustáceos decápodos (thalassínidos o callianássidos).

\section{Contexto paleoambiental}

Gyrolithes suele ser indicativo de ambientes marinos marginales (ver Gernant, 1972, p. 738) litorales de aguas salobres (Beynon et al., 1988), aunque también se han registrado en menor medida en ambientes mucho más profundos [rampa carbonatada externa por debajo del nivel de base del oleaje en el Jurásico (Pliensbachiense) de Asturias, García Ramos, comunicación personal] e incluso continentales terrestres. En nuestro caso, Gyrolithes nodosus nov. ichnosp. se encuentra en los términos transgresivos inferiores de la serie del Plioceno Inferior, concretamente, en la parte baja del conjunto limo-arenoso. Esta parte se sitúa entre los niveles submareales del tramo 1 y los niveles que presentan las superficies diastémicas y lumaquélicas, de batimetría similar o incluso intermareal, del tramo 4 (Mayoral y Muñiz, 1994, 1995), por lo que podría situarse en un medio de características paleoambientales semejantes.

\section{CONCLUSIONES}

El estudio de las características morfológicas en nuevos ejemplares de Gyrolithes permite reconocer la existencia de una pared que presenta un revestimiento acusado, de carácter aglutinante. Éste está constituido por un conjunto de pelets limo-arenosos que se disponen de forma homogénea cubriendo totalmente la estructura.

Su conexión directa con Ophiomorpha nodosa es un criterio más para reconocer la singularidad de estos ejemplares y justificar su propuesta como una icnoespecie nueva: Gyrolithes nodosus nov. ichnosp.

\section{AGRADECIMIENTOS}

A D. José Antonio Gámez Vintaned y al Dr. Eladio Liñán del Departamento de Geología (Área de Paleontología) de la Universidad de Zaragoza; al Dr. J.C. García-Ramos del Departamento de Geología (Área de Estratigrafía) de la Universidad de Oviedo y a D. Mariano Verde del Departamento de Paleontología (Facultad de Ciencias) de la Universidad de la República en Montevideo (Uruguay) por la información suministrada y los comentarios realizados, que han mejorado la calidad final de este artículo. Este trabajo se ha realizado dentro del marco del Grupo de Investigación n RNM 0219 "G.I. NATURALAND" de la Junta de Andalucía, de los Proyectos de la D.G.I.C.Y.T. PB-94-0946 y de la Acción Integrada Hispano-Portuguesa HP-9546.

\section{BIBLIOGRAFÍA}

Barbour, I. H. 1892. Note on new gigantic fossils. Science, 99-100.

Beynon, B.M., Pemberton, S.G., Bell, D.D. and Logan, C.A. 1988. Environmental implications of ichnofossils from the Lower Cretaceous Grand Rapids Formation; Cold Lake Oil Sands Deposit. In: Sequences, Stratigraphy, Sedimentology: Surface and Subsurface. (Eds. D.P. James and D.A. Leckie). Canadian Society of Petroleum Geologists, Memoir 15, 275-290.

Bromley, R. G. 1990. Trace Fossils: Biology and Taphonomy. Unwin Hyman, London, 280 pp.

Bromley, R. G. and Frey, R. W. 1974. Redescription of the trace fossil Gyrolithes and taxonomic evaluation of Thalassinoides, Ophiomorpha and Spongeliomorpha. Geological Society of Denmark Bulletin, 23, 311-335.

Cáceres Puro, L. M. 1995. Geomorfología del sector occidental de la Depresión del Guadalquivir. Tesis Doctoral (inédita). Universidad de Huelva, 292 pp.

Christiansen, J. C. and Curran, H. A. 1995. The trace fossil Gyrolithes: unwinding the spiral enigma in the St. Marys Formation (Miocene) of Maryland. Geological Society of America, Abstracts with Programs, Norteastern Section, 27, 35-36.

Dryden, L. 1933. Xenohelix in the Maryland Miocene. Natur Academy of Science Proceeding, 19, 139-143.

Dworschak, P. C. and Rodrigues, S. 1997. A modern analogue for the trace fossil Gyrolithes: burrows of the thalassinidean shrimp Axianassa australis. Lethaia, 30, 41-52.

Ekdale, A. A. 1992. Muckraking and Mudslinging: the Joys of Deposit-Feeding. In: Trace Fossils (Eds. C. G. Mapples \& R. R. West). Short Courses in Paleontology, 5, A Publication of Paleontological Asociation, 145-171.

Ekdale, A. A., Bromley, R. G. and Pemberton, S. G., 1984. Ichnology. The use of trace fossils in sedimentology and stratigraphy. Society of Economic Paleontologists and Mineralogists, Short Course 15, 1-317.

Fedonkin, M. A. 1980. Rannie etapi evoliutsii Metazoa po paleoichnologicheskim dannim. Akademiya Nauk SSSR. Seriya Geologicheskaya, 2, 226-233.

Föllmi, K. B. and Grimm, K. A. 1990. Doomed pioneers: Gravity-flow deposition and bioturbation in marine oxygen-deficient enviroments. Geology, 18, 1069-1072.

Frey, R. W., Howard, J. and Pryor, W. A. 1978. Ophiomorpha: its morphologic, taxonomic and enviromental significance. Palaeogeography, Palaeoclimatology, Palaeoecology, 23, 199-299.

Gámez Vintaned, J. A. 1995. Los materiales prehercínicos de la Sierra del Moncayo (Cadena Ibérica Occidental, España) y su contenido paleoicnológico. Boletín de la Real Sociedad Española de Historia Natural (Sección Geológica), 90, 21-55.

Gámez Vintaned, J. A. y Liñán, E. 1996. Revisión de la terminología icnológica en español. Revista Española de Paleontología, 11, 155-176.

Gernant, R. E. 1970. Paleoecology of the Choptank Formation (Miocene) of Maryland and Virginia. Maryland Geological Survey, Rept. Inv., 12, 90.

Gernant, R.E. 1971. Invertebrate biofacies and paleoenvironments. In: Environmental history of the Maryland Miocene. Maryland Geological Survey. Guidebook, 3, 19-30.

Gernant, R. E. 1972. The paleoenviromental significance of Gyrolithes (Lebensspur). Journal of Paleontology, 46, 735-741. 
Häntzschel, W. 1934. Schraubenförmige und spiralige GrabGänge in Turonen Sandsteinen des Zittauer Gebirges. Abhandlungen Senckenbergsche Naturforschenda Geselschaft, 429, 313-324.

Häntzschel, W. 1962. Trace Fossils and Problematica. In: Treatise on Invertebrate Paleontology (Ed. R. C. Moore). Geological Society of America \& University of Kansas Press. New York \& Lawrence, Kansas, 177-245.

Häntzschel, W. 1965. Vestigia Invertebratorum et Problematica. Fossilium catalogous. 1: Animalia, Pars 108, 1-142.

Hester, N. C. and Pryor, W. A. 1972. Blade-shaped crustaceans burrows of Eocene age: a composite form of Ophiomorpha. Bulletin of the Geological Society of America, 83, 677-688.

Horst, C. J. van der. 1940. The Enteropneusta from Inyack Island, Delagou Bay. South African Museum Annals, 32, 293-380.

Keij, A. J. 1965. Miocene trace fossils from Borneo. Paläntologische Zeitschrift, 39, 220-228.

Kilpper, K. 1962. Xenohelix Mansfield 1927 aus der miozänen niederr Heinischen Braunkihlenformation. Paläntologische Zeitschrift, 36 (7), 55-58.

King, M. M. 1987. Infaunal tiering in tidal channel deposits of the Eocene Delmar Formation, Solana Beach and Torrey Pines State Reserve, Southern California. In: New Concepts in the use of biogenic sedimentary structures for paleoenvironmental interpretation (Ed. D. J. Bottjer). The Pacifics Section Society of Economic Paleontologists and Mineralogists, 52, 35- 40.

Liñán, E. 1984. Los Icnofósiles de la Formación Torreárboles (Precámbrico?-Cámbrico inferior) en los alrededores de Fuente de Cantos, Badajoz. Cuadernos do Laboratorio Xeologico do Laxe, 8, 47-74.

Macsotay, O. 1967. Huellas problemáticas y su valor paleoecológico en Venezuela, GEOS (Venezuela), 16, 7-79.

Mansfield, W. C. 1927. Some pecculiar fossils from Maryland. Proceedings of the United States National Museum, 71, 1-9.

Mansfield, W. C. 1930. Some pecculiar fossils forms from California and Mexico. Proceedings of the United States National Museum, 77, 1-3.

Mapples, Ch. G. and West, R. R. 1992. Trace Fossils. Short Courses in Paleontology, 5. A Publication of the Paleontological Association, 1-238.

Mayoral, E. 1986. Gyrolithes vidali nov. icnoesp. (Plioceno marino) en el sector Suroccidental de la Cuenca del Guadalquivir. Estudios Geológicos, 42, 211-223.

Mayoral, E. y Muñiz, F. 1993. Consideraciones paleoetológicas acerca de Gyrolithes. Comunicaciones de las IX Jornadas de Paleontología, Málaga, 18-22.
Mayoral, E. y Muñiz, F. 1994. Presencia de un nuevo Cefalópodo sepioideo en el Mioceno Superior de la Cuenca del Guadalquivir (Lepe, Huelva; España). Coloquios de Paleontología, 46, 161-174.

Mayoral, E. y Muñiz, F. 1995. Nueva icnoespecie de Gyrolithes del Mioceno Superior de la Cuenca del Guadalquivir (Lepe, Huelva). Revista Española de Paleontología, 10, 190-201.

Mayoral, E. y Muñiz, F. 1996. La icnofacies de Gnathichnus en el sector suroccidental de la Cuenca del Guadalquivir (Lepe, Huelva, España). Coloquios de Paleontología, 48, 87-102.

Muñiz, F. y Mayoral, E. 1996. Nuevos datos icnotaxonómicos sobre Gyrolithes del Mioceno superiorPlioceno? de la Cuenca del Guadalquivir (Lepe, Huelva). Comunicaciones de las XII Jornadas de Paleontología, Badajoz, 30 de octubre-2 de noviembre de 1996, Universidad de Extremadura, Servicio de Publicaciones, Cáceres, 86-88.

Muñiz, F., Mayoral, E. y Martín, M. 1995. Pistas fósiles intergradacionales en el Mioceno superior del sector suroccidental de la Cuenca del Guadalquivir. Comunicaciones de las XI Jornadas de Paleontología, Tremp (Lérida), 119-122.

Okada, H. and Bukry, D. 1980. Supplementary modification and introduction of code numbers to the low-latitude coccolith biostratigraphic zonation (Bukry, 1973, 1979). Marine Micropaleontology, 5(3), 171-187.

Powell, E. N. 1977. The relationship of the trace fossil Gyrolithes (=Xenohelix) to the family Capitellidae (Polychaete). Journal of Paleontology, 51, 552-556.

Saporta, L.C.J.G. de. 1884. Les organismes problematiques des anciennes mers. Masson, Paris. 100 pp.

Schultz, C. B. 1942. A review of the Daimonhelix problem. University of Nebraska Studies, Studies in Science and Technology, 2, 1-30.

Stanton, R. J. Jr. and Warme, J. E. 1971. Stop 1: Stone City Bluff. In : Trace Fossils. A Field Guide to Selected Localities in Pennsilvanian, Permian, Cretaceous and Terciary rocks of Texas and Related Papers. (Ed. B. F. Perkins). School of Geoscience Louisiana State University Miscellaneous Publication, 71(1), 3-10.

Stenzel, H. B., Krause, E.K. and Twining, J. T. 1957. Pelecypoda from the type locality of the Stone City Beds (Middle Eocene) of Texas. University of Texas, Publication. 5704, 237.

Toots, H. 1963. Helical burrows as fossils movement patterns. Contributions of Geology, 2, 129-134.

Woodward, B. B. 1922. On Dinocochlea ingens n. g. n. sp., a gigantic gastropod from the Wealden Beds near Hastings. Geological Magazine, 59, 242-248. 